ANL-HEP-PR-97-01

\title{
Threshold Resummation of the Total Cross Section for Heavy Quark Production in Hadronic Collisions
}

\author{
Edmond L. Berger and Harry Contopanagos* \\ High Energy Physics Division, Argonne National Laboratory \\ Argonne, Illinois 60439, USA
}

(May 26, 1997)

\begin{abstract}
We discuss calculations of the inclusive total cross section for heavy quark production at hadron collider energies within the context of perturbative quantum chromodynamics, including resummation of the effects of initial-state soft gluon radiation to all orders in the strong coupling strength. We resum the universal leading-logarithm contributions, and we restrict our integrations to the region of phase space that is demonstrably perturbative. We include a detailed comparison of the differences between ours and other methods. We provide predictions of the physical cross section as a function of the heavy quark mass in proton-antiproton reactions at center-of-mass energies of 1.8 and 2.0 TeV, and we discuss estimated uncertainties.
\end{abstract}

Typeset using REVTEX

*Current address: Electrical Engineering Department, University of California at Los Angeles, Los Angeles, CA 90024 


\section{INTRODUCTION AND MOTIVATION}

In this report we present and discuss calculations carried out in perturbative quantum chromodynamics (QCD) of the inclusive cross section for the production of heavy quarkantiquark pairs in hadron reactions [1 1 . In most of the paper, we identify the heavy quark as a top quark, $t$, but the results are valid more generally as long at the mass of the quark is sufficiently heavy. For example, they should apply as well to production of a fourth-generation quark, such as a postulated $b^{\prime}$.

In inclusive hadron interactions at collider energies, $h_{1}+h_{2} \rightarrow t+\bar{t}+X, t \bar{t}$ pair production proceeds through partonic hard-scattering processes involving initial-state light quarks $q$ and gluons $g$. In lowest-order QCD, at $\mathcal{O}\left(\alpha_{s}^{2}\right)$, the two partonic subprocesses are $q+\bar{q} \rightarrow t+\bar{t}$ and $g+g \rightarrow t+\bar{t}$. Calculations of the cross section through next-to-leading order, $\mathcal{O}\left(\alpha_{s}^{3}\right)$, involve gluonic radiative corrections to these lowest-order subprocesses as well as contributions from the $q+g$ initial state [5]. A complete fixed-order calculation at order $\mathcal{O}\left(\alpha_{s}^{n}\right), n \geq 4$ does not exist. In this paper, we do not examine mechanisms for the production of single top quarks or antiquarks [6].

The physical cross section for each production channel is obtained through the factorization theorem,

$$
\sigma_{i j}\left(S, m^{2}\right)=\frac{4 m^{2}}{S} \int_{0}^{\frac{S}{4 m^{2}}-1} d \eta \Phi_{i j}\left[\frac{4 m^{2}}{S}(1+\eta), \mu^{2}\right] \hat{\sigma}_{i j}\left(\eta, m^{2}, \mu^{2}\right)
$$

The square of the total hadronic center-of-mass energy is $S$, and the square of the partonic center-of-mass energy is $s$. The mass of the heavy quark is $m$, and $\mu$ is the common renormalization/factorization scale of the problem. The variable $\eta=\frac{s}{4 m^{2}}-1$ measures the distance from the partonic threshold. The indices $i j \in\{q \bar{q}, g g\}$ denote the initial parton channel. The partonic cross section $\hat{\sigma}_{i j}\left(\eta, m^{2}, \mu^{2}\right)$ is obtained commonly from fixed-order QCD calculations [5], or, as described here, from calculations that go beyond fixed-order perturbation theory through the inclusion of gluon resummation [1]. The parton flux is $\Phi_{i j}\left(y, \mu^{2}\right)=\int_{y}^{1} \frac{d x}{x} f_{i / h_{1}}\left(x, \mu^{2}\right) f_{j / h_{2}}\left(y / x, \mu^{2}\right)$, where $f_{i / h_{1}}\left(x, \mu^{2}\right)$ is the density of partons of 
type $i$ in hadron $h_{1}$. We use the notation $\alpha(\mu) \equiv \alpha_{s}(\mu) / \pi$. Unless otherwise specified, $\alpha \equiv \alpha(\mu=m)$ throughout this paper. The total physical cross section is obtained after incoherent addition of the contributions from the the $q \bar{q}$ and $g g$ production channels. In this paper, we ignore the small contribution from the $q g$ channel.

Comparison of the partonic cross section at next-to-leading order with its lowest-order value reveals that the ratio becomes very large in the near-threshold region. Indeed, as $\eta \rightarrow 0$, the "K-factor" at the partonic level $\hat{K}(\eta)$ grows in proportion to $\alpha \ln ^{2}(\eta)$. An illustration of this behavior may be seen in Fig. 7 of Ref. [3]. The very large mass of the top quark notwithstanding, the large ratio $\hat{K}(\eta)$ makes it evident that the next-to-leading order result does not necessarily provide a trustworthy quantitative prediction of the top quark production cross section at the energy of the Tevatron collider. The large ratio casts doubt on the reliability of simple fixed-order perturbation theory for physical processes for which the near-threshold region in the subenergy variable contributes significantly to the physical cross section. Top quark production at the Fermilab Tevatron is one such process, because the top mass is relatively large compared to the energy available. Other examples include the production of hadronic jets that carry large values of transverse momentum and the production of pairs of supersymmetric particles with large mass. To obtain more dependable theoretical estimates of the cross section in perturbative QCD, it is important first to identify and isolate the terms that provide the large next-to-leading order enhancement and then to resum these effects to all orders in the strong coupling strength.

We begin in Sec. II with the motivation for the inclusion of the effects of intial state soft gluon radiation to all orders in the QCD coupling strength, and we review the general formalism of resummation. In Sec. III, we outline the method and domain of applicability of perturbative resummation that we developed in the past year [2,3]. We present predictions in Sec. IV of the physical cross section as a function of the heavy quark mass in protonantiproton reactions at center-of-mass energies of 1.8 and $2.0 \mathrm{TeV}$, and we discuss estimated uncertainties. Our calculation is in good agreement with the measured cross section at the reported mass of the top quark [0]. At $m=175 \mathrm{GeV}$ and $\sqrt{S}=1.8 \mathrm{TeV}$, the all-orders 
resummed cross section is about $9 \%$ greater than the next-to-leading order value. Since the large threshold logarithms are mastered by resummation, the theoretical reliability of the resummed result is considerably greater than that of the fixed order calculation. At other values of $m$ and $\sqrt{S}$, where the ratio $m / \sqrt{S}$ is larger, the numerical effects of resummation can be more significant. In Secs. V and VI, we compare our approach and results with other methods [1, 4] and address criticisms that have been made [幽. The difference between our approach and that of Ref. [4] resides in the treatment of subleading logarithmic contributions, and we explain our reasons for preferring our method. Conclusions are summarized in Sec. VII.

\section{GLUON RADIATION AND RESUMMATION}

The origin of the large threshold enhancement may be traced to initial-state gluonic radiative corrections to the lowest-order channels. To avoid misunderstanding, we remark that we are calculating the inclusive total cross section for the production of a top quarkantiquark pair, i.e., the total cross section for $t+\bar{t}+$ anything. The partonic subenergy threshold in question is the threshold for $t+\bar{t}+$ any number of gluons. This coincides with the threshold in the invariant mass of the $t+\bar{t}$ system for the lowest order subprocesses only.

To specify the kinematic variables, we consider the two-to-three parton subprocess $i\left(k_{1}\right)+$ $j\left(k_{2}\right) \rightarrow t\left(p_{1}\right)+\bar{t}\left(p_{2}\right)+g(k)$. We define the variable $z$ through the partonic invariants [1]

$$
s=\left(k_{1}+k_{2}\right)^{2}, t_{1}=\left(k_{2}-p_{2}\right)^{2}-m^{2}, u_{1}=\left(k_{1}-p_{2}\right)^{2}-m^{2},(1-z) m^{2}=s+t_{1}+u_{1} .
$$

Alternatively, $(1-z)=\frac{2 k \cdot p_{1}}{m^{2}}$. In the limit that $z \rightarrow 1$, the radiated gluon $g(k)$ carries zero momentum. After cancellation of soft singularities and factorization of collinear singularities in $\mathcal{O}\left(\alpha^{3}\right)$, there are left-over integrable logarithmic contributions to the cross section associated with initial-state gluon radiation. The contributions of interest here, often expressed in terms of "plus" distributions, are proportional to $\ln (1-z)$. These logarithmic terms are vestiges of the canceled infrared singularities. 
The partonic cross section may be expressed generally as

$$
\hat{\sigma}_{i j}\left(\eta, m^{2}\right)=\int_{z_{\min }}^{1} d z \mathcal{H}_{i j}(z, \alpha) \hat{\sigma}_{i j}^{\prime}\left(\eta, m^{2}, z\right) .
$$

We work in the $\overline{\mathrm{MS}}$ factorization scheme in which the $q, \bar{q}$ and $g$ densities and the next-to-leading order partonic cross sections are defined unambiguously. The lower limit of integration, $z_{\min }=1-4(1+\eta)+4 \sqrt{1+\eta}$, is set by kinematics. The derivative $\hat{\sigma}_{i j}^{\prime}\left(\eta, m^{2}, z\right)=d\left(\hat{\sigma}_{i j}^{(0)}\left(\eta, m^{2}, z\right)\right) / d z$, and $\hat{\sigma}_{i j}^{(0)}$ is the lowest-order $\mathcal{O}\left(\alpha^{2}\right)$ partonic cross section expressed in terms of inelastic kinematic variables to account for the emitted radiation.

Keeping only the leading logarithmic contributions through $\mathcal{O}\left(\alpha^{3}\right)$, we may approximate the total partonic cross section as

$$
\begin{aligned}
\hat{\sigma}_{i j}^{(0+1)}\left(\eta, m^{2}\right) & =\int_{z_{\min }}^{1} d z\left\{1+\alpha 2 C_{i j} \ln ^{2}(1-z)\right\} \hat{\sigma}_{i j}^{\prime}\left(\eta, z, m^{2}\right) \\
& \equiv \int_{z_{\min }}^{1} d z \mathcal{H}_{i j}^{(0+1)}(z, \alpha) \hat{\sigma}_{i j}^{\prime}\left(\eta, z, m^{2}\right)
\end{aligned}
$$

where $C_{q \bar{q}}=C_{F}=4 / 3$ and $C_{g g}=C_{A}=3$. As is illustrated in Fig. 1, the leading logarithmic contribution, integrated over the near-threshold region $1 \geq z \geq 0$, provides an excellent approximation to the exact full next-to-leading order physical cross section as a function of the heavy quark mass.

Although a fixed-order $\mathcal{O}\left(\alpha^{4}\right)$ calculation of $t \bar{t}$ pair production does not exist, we may invoke universality with massive lepton-pair production $(l \bar{l})$, the Drell-Yan process, to generalize Eq. (4) to higher order. In the near-threshold region, the hard kernel becomes

$$
\mathcal{H}_{i j}^{(0+1+2)}(z, \alpha) \simeq 1+2 \alpha C_{i j} \ln ^{2}(1-z)+\alpha^{2}\left[2 C_{i j}^{2} \ln ^{4}(1-z)-\frac{4}{3} C_{i j} b_{2} \ln ^{3}(1-z)\right] .
$$

The coefficient $b_{2}=\left(11 C_{A}-2 n_{f}\right) / 12$, and the number of flavors $n_{f}=5$. We note that the leading logarithmic contributions in each order of perturbation theory are all positive in overall sign [8] so that the leading logarithm threshold enhancement keeps building in magnitude at each fixed order of perturbation theory. The further enhancement of the physical cross section produced by the $\mathcal{O}\left(\alpha^{4}\right)$ leading logarithmic terms in the nearthreshold region is shown in Fig. 1. At $m=175 \mathrm{GeV}$, we compute the following ratios of 
the physical cross sections in the leading logarithmic approximation: $\sigma_{i j}^{(0+1)} / \sigma_{i j}^{(0)}=1.22$, and $\sigma_{i j}^{(0+1+2)} / \sigma_{i j}^{(0+1)}=1.14$.

The goal of gluon resummation is to sum the series in $\alpha^{n} \ln ^{2 n}(1-z)$ to all orders in $\alpha$ in order to obtain a more trustworthy prediction. This procedure has been studied extensively for the Drell-Yan process [9], and good agreement with data is achieved. In essentially all resummation procedures, the large logarithmic contributions are exponentiated into a function of the QCD running coupling strength, itself evaluated at a variable momentum scale that is a measure of the radiated gluon momentum. For example, in the approach of Laenen, Smith, and van Neerven (LSvN) [1], the resummed partonic cross section is written as

$$
\hat{\sigma}_{i j}^{R ; I R C}\left(\eta, \mu_{o}\right)=\int_{z_{m i n}}^{1-\left(\mu_{o} / m\right)^{3}} d z \mathrm{e}^{E_{i j}\left(z, m^{2}\right)} \hat{\sigma}_{i j}^{\prime}\left(\eta, m^{2}, z\right),
$$

where the exponent

$$
E_{i j}\left(z, m^{2}\right) \propto C_{i j} \alpha\left((1-z)^{2 / 3} m^{2}\right) \ln ^{2}(1-z) .
$$

We note that in Eq. (17), the strong coupling strength is evaluated at the variable momentum scale $(1-z)^{2 / 3} m^{2}$.

Different methods of resummation differ in theoretically and phenomenologically important respects. The set of purely leading monomials $\alpha^{n} \ln ^{2 n}(1-z)$ in $\hat{\sigma}_{i j}$ exponentiates directly, with $\alpha$ evaluated at a fixed large scale $\mu=m$, as may be appreciated from a glance at Eq. (5). This simple result does not mean that a theory of resummation is redundant, even if only leading logarithms are to be resummed. Indeed, straightforward replacement of the term within the brackets of Eq. (4) with the exponential of $\alpha 2 C_{i j} \ln ^{2}(1-z)$ would lead to an exponentially divergent integral (and therefore cross section) since the coefficient of the logarithm is positive. The naive approach, therefore, fails from the start, and more sophisticated resummation approaches must be employed, involving scaling and Lorentztransformation properties of the classes of terms to be summed. The more sophisticated approaches are not free from problems, however. Formally, if not explicitly in some approaches, an integral over the radiated gluon momentum $z$ must be done over regions in 
which $z \rightarrow 0$. Therefore, one significant distinction among methods has to do with how the inevitable "non-perturbative" region is handled in each case. Examination of Eqs. (6) and (7) shows that an infrared singularity is encountered in the soft-gluon limit $z \rightarrow 1$ : owing to the logarithmic behavior of $\alpha\left(q^{2}\right), \alpha\left(q^{2}\right) \propto \ln ^{-1}\left(q^{2} / \Lambda_{Q C D}^{2}\right), \alpha\left((1-z)^{2 / 3} m^{2}\right) \rightarrow \infty$ as $z \rightarrow 1$. The infrared singularity is a manifestation of non-perturbative physics. In the approach of LSvN, this divergence of the integrand at the upper limit of integration necessitates introduction of the undetermined infrared cutoff (IRC) $\mu_{o}$ in Eq. (6), with $\Lambda_{Q C D} \leq \mu_{o} \leq m$. The cutoff prevents the integration over $z$ from reaching the Landau pole of the QCD running coupling constant. The presence of an extra scale spoils the renormalization group properties of the overall expression. The unfortunate dependence of the resummed cross section on this undetermined cutoff is important numerically since it appears in an exponent [1]. Theoretical uncertainties are not easy to evaluate quantitatively in a method that relies on an undetermined infrared cutoff.

\section{PERTURBATIVE RESUMMATION}

The method of resummation we employ [2,3] is based on a perturbative truncation of principal-value resummation (PVR). The principal-value method [9] has an important technical advantage in that it does not require arbitrary infrared cutoffs, as all Landau-pole singularities are by-passed by a Cauchy principal-value prescription. Because extra undetermined scales are absent, the method also permits an evaluation its perturbative regime

of applicability, i.e., the region of the gluon radiation phase space where resummed perturbation theory should be valid.

To illustrate how infrared cutoffs are avoided in the PVR method, it is useful to begin with an expression in moment $(n)$ space for the exponent that resums the $\ln (1-z)$ terms [10]. Factorization and evolution lead directly to exponentiation in moment space:

$$
E\left(n, m^{2}\right)=-\int_{0}^{1} d x \frac{x^{n-1}-1}{1-x} \int_{(1-x)^{2}}^{1} \frac{d \lambda}{\lambda} g\left[\alpha\left(\lambda m^{2}\right)\right] \text {. }
$$


The function $g(\alpha)$ is calculable perturbatively, but the behavior of $\alpha\left(\lambda m^{2}\right)$ leads to a divergence of the integrand when $\lambda m^{2} \rightarrow \Lambda_{Q C D}^{2}$. To tame the divergence, a cutoff can be introduced in the integral over $x$ or directly in momentum space, in the fashion of LSvN. In the principal-value redefinition of resummation, the singularity is avoided by replacement of the integral over the real axis $x$ in Eq. (8) by an integral in the complex plane along a contour $P$ that has the same endpoints and is symmetric under reflections across the real axis:

$$
E^{P V}\left(n, m^{2}\right) \equiv-\int_{P} d \zeta \frac{\zeta^{n-1}-1}{1-\zeta} \int_{(1-\zeta)^{2}}^{1} \frac{d \lambda}{\lambda} g\left[\alpha\left(\lambda m^{2}\right)\right]
$$

The function $E^{P V}\left(n, m^{2}\right)$ is finite since the Landau pole singularity is by-passed. Moreover, $\lim _{n \rightarrow \infty} E^{P V}\left(n, m^{2}\right)=-\infty$, and, therefore, the corresponding partonic cross section is finite as $z \rightarrow 1(n \rightarrow+\infty)$. In Eq. (9), all large soft-gluon threshold contributions are included through the two-loop running of $\alpha$.

Equations (8) and (9) have identical perturbative content, but they have different nonperturbative content since the infrared region is treated differently in the two cases. The non-perturbative content is not a prediction of perturbative QCD. In our study of top quark production, we choose to use the exponent only in the region of phase space in which the perturbative content dominates.

We use the attractive finiteness of Eq. (9) to derive a perturbative asymptotic representation of $E(x, \alpha(m))$ that is valid in the moment-space interval

$$
1<x \equiv \ln n<t \equiv \frac{1}{2 \alpha b_{2}} .
$$

This perturbative asymptotic representation is

$$
E_{i j}(x, \alpha) \simeq E_{i j}(x, \alpha, N(t))=2 C_{i j} \sum_{\rho=1}^{N(t)+1} \alpha^{\rho} \sum_{j=0}^{\rho+1} s_{j, \rho} x^{j}
$$

Here

$$
s_{j, \rho}=-b_{2}^{\rho-1}(-1)^{\rho+j} 2^{\rho} c_{\rho+1-j}(\rho-1) ! / j !,
$$


and $\Gamma(1+z)=\sum_{k=0}^{\infty} c_{k} z^{k}$, where $\Gamma$ is the Euler gamma function. The number of perturbative terms $N(t)$ in Eq. (11) is obtained [3] by optimizing the asymptotic approximation

$$
|E(x, \alpha)-E(x, \alpha, N(t))|=\text { minimum. }
$$

Because of the range of validity in Eq. (10) and owing to the optimization Eq. (13), terms in the exponent of the form $\alpha^{k} \ln ^{k} n$ are of order unity, and terms with fewer powers of logarithms, $\alpha^{k} \ln ^{k-m} n$, are negligible. The optimization assures us that the coefficients of the various terms are benign. Resummation is completed in a finite number of steps. With a two-loop expression for the running coupling strength, all monomials of the form $\alpha^{k} \ln ^{k+1} n, \alpha^{k} \ln ^{k} n$ are produced in the exponent of Eq. (11). Because of the restricted leading-logarithm universality between the $t \bar{t}$ and $l \bar{l}$ processes, we discard monomials of the form $\alpha^{k} \ln ^{k} n$ in the exponent.

The exponent we use is the truncation

$$
E_{i j}(x, \alpha, N)=2 C_{i j} \sum_{\rho=1}^{N(t)+1} \alpha^{\rho} s_{\rho} x^{\rho+1},
$$

with the coefficients $s_{\rho} \equiv s_{\rho+1, \rho}=b_{2}^{\rho-1} 2^{\rho} / \rho(\rho+1)$. The number of perturbative terms $N(t)$ is a function of only the top quark mass $m$. This expression contains no factorially-growing (renormalon) terms. The perturbative region of phase space is far removed from the part of phase space in which renormalons could be influential.

In Fig. 2 we illustrate the validity of the asymptotic approximation for a value of $t$ corresponding to $m=175 \mathrm{GeV}$. Optimization works perfectly, with $N(t)=6$, and the plot demonstrates the typical breakdown of the asymptotic approximation if $N$ is allowed to increase beyond $N(t)$. This rise represents the exponential growth of the infrared (IR) renormalons, the $(\rho-1)$ ! growth in the second term of Eq. (12). As long as $n$ is in the interval of Eq. (10), all the members of the family in $n$ are optimized at the same $N(t)$, showing that the optimum number of perturbative terms is a function of $t$, i.e., of $m$ only.

It is valuable to stress that we can derive the perturbative expressions, Eqs. (10), (11), and (12), from the unregulated exponent Eq. (8) without the PVR prescription, although with less certitude. We discuss this point in some detail in Sec. III.B of our long paper [3]. 
After inversion of the Mellin transform from moment space to the physically relevant momentum space, the resummed partonic cross sections, including all large threshold corrections, can be written in the form of Eq. (3), but with the hard kernel replaced by the resummed form

$$
\mathcal{H}_{i j}^{R}(z, \alpha)=\int_{0}^{\ln \left(\frac{1}{1-z}\right)} d x \mathrm{e}^{E_{i j}(x, \alpha)} \sum_{j=0}^{\infty} Q_{j}(x, \alpha)
$$

The leading large threshold corrections are contained in the exponent $E_{i j}(x, \alpha)$, a calculable polynomial in $x$. The functions $\left\{Q_{j}(x, \alpha)\right\}$ arise from the analytical inversion of the Mellin transform from moment space to momentum space. These functions are produced by the resummation and are expressed in terms of successive derivatives of $E$ : $P_{k}(x, \alpha) \equiv \partial^{k} E(x, \alpha) / k ! \partial^{k} x$. Each $Q_{j}$ contains $j$ more powers of $\alpha$ than of $x$ so that Eq. (15) embodies a natural power-counting of threshold logarithms.

The functional form of $E_{i j}$ for $t \bar{t}$ production is identical to that for $l \bar{l}$ production, except for the identification of the two separate channels, denoted by the subscript $i j$. However, only the leading threshold corrections are universal. Final-state gluon radiation as well as initial-state/final-state interference effects produce subleading logarithmic contributions that differ for processes with different final states. Accordingly, there is no physical basis for accepting the validity of the particular subleading terms that appear in Eq. (15). Among all $\left\{Q_{j}\right\}$ in Eq. (15), only the very leading one is universal. This is the linear term in $P_{1}$ contained in $Q_{0}$, that turns out to be $P_{1}$ itself. Since we intend to resum only the universal leading logarithms, we retain only $P_{1}$. Hence, Eq. (15) can be integrated explicitly, and the resummed version of Eq. (3) is

$$
\hat{\sigma}_{i j}^{R ; p e r t}\left(\eta, m^{2}\right)=\int_{z_{\min }}^{z_{\max }} d z \mathrm{e}^{E_{i j}\left(\ln \left(\frac{1}{1-z}\right), \alpha\right)} \hat{\sigma}_{i j}^{\prime}\left(\eta, m^{2}, z\right)
$$

We have inserted an upper limit of integration, $z_{\max }$, in Eq. (16). This upper limit is set by the boundary between the perturbative and non-perturbative regimes. An intuitive definition of the perturbative region, where inverse power terms are unimportant, is provided by the inequality $\frac{\Lambda_{Q C D}}{(1-z) m} \leq 1$. This inequality is identical to the expression in moment space, 
Eq. (10), with the identification $n=\frac{1}{1-z}$. In momentum space, the same condition is realized by the constraint that all $\left\{Q_{j}\right\}, j \geq 1$ be small compared to $Q_{0}$. From the explicit expressions [3] for the $\left\{Q_{j}\right\}$, one may show that this constraint corresponds to

$$
P_{1}\left(\ln \left(\frac{1}{1-z}\right), \alpha\right) \leq 1
$$

Equation (17) is equivalent to the requirement that terms that are subleading according to perturbative power-counting are indeed subleading numerically; Eq. (17) is the essence of perturbation theory in this context. It assures us that our integration is carried out only over a range in which poorly specified subleading terms would not contribute significantly even if they had been retained.

As remarked above, we accept only the perturbative content of principal-value resummation, and our cross section is evaluated accordingly. Specifically, we use Eq. (16) with the upper limit of integration, $z_{\max }$, calculated from Eq. (17). The upshot is an effective threshold boundary on the integral over the scaled subenergy variable $\eta$, but one that is calculable, not arbitrary. While reminiscent perhaps of the cutoff used in the LSvN approach, our threshold boundary has a very different and well defined origin. Our perturbative resummation probes the threshold down to the point $\eta \geq \eta_{0}=\left(1-z_{\max }\right) / 2$. Below this value, perturbation theory, resummed or otherwise, is not to be trusted. For a top mass $m$ $=175 \mathrm{GeV}$, we determine that the perturbative regime is restricted to $\eta \geq 0.007$ for the $q \bar{q}$ channel and $\eta \geq 0.05$ for the $g g$ channel. These numbers may be converted to more readily understood values of the subenergy above which we judge our perturbative approach is valid: at $m=175 \mathrm{GeV}$, these are $1.22 \mathrm{GeV}$ above the threshold in the $q \bar{q}$ channel, and $8.64 \mathrm{GeV}$ above the threshold in the $g g$ channel. The difference reflects the larger color factor in the $g g$ case. A larger color factor makes the non-perturbative region larger. (One could attempt to apply Eq. (16) all the way to $z_{\max }=1$, i.e., to $\eta=0$, but one would then be using a model for non-perturbative effects, the one suggested by PVR, below the region justified by perturbation theory.) We note that the value $1.22 \mathrm{GeV}$ in the $q \bar{q}$ channel is comparable to the decay width of the top quark, $\Gamma\left(t \rightarrow b W^{+}\right)=1.55 \mathrm{GeV}$. The width itself 
provides a natural definition of the minimum non-perturbative region. The two independent determinations of the non-perturbative region are in agreement [11].

\section{PHYSICAL CROSS SECTION}

In order to achieve the best accuracy available we wish to include in our predictions as much as is known theoretically. Our final resummed partonic cross section can therefore be written [2,3]

$$
\hat{\sigma}_{i j}^{\text {pert }}\left(\eta, m^{2}, \mu^{2}\right)=\hat{\sigma}_{i j}^{R ; p e r t}\left(\eta, m^{2}, \mu^{2}\right)-\left.\hat{\sigma}_{i j}^{(0+1)}\left(\eta, m^{2}, \mu^{2}\right)\right|_{R ; p e r t}+\hat{\sigma}_{i j}^{(0+1)}\left(\eta, m^{2}, \mu^{2}\right) .
$$

The second term is the part of the partonic cross section up to one-loop that is included in the resummation, while the last term is the exact one-loop cross section [5]. To obtain physical cross sections, we insert Eq. (18) into Eq. (1), and we integrate over $\eta$. Other than the heavy quark mass, the only undetermined scales are the QCD factorization and renormalization scales. We adopt a common value $\mu$ for both, and we vary this scale over the interval $\mu / m \in\{0.5,2\}$ in order to evaluate the theoretical uncertainty of the numerical predictions. We use the CTEQ3M parton densities [12].

A quantity of phenomenological interest is the differential cross section $\frac{d \sigma_{i j}\left(S, m^{2}, \eta\right)}{d \eta}$. Its integral over $\eta$ is the total cross section. In Fig. 3 we plot these distributions for $m=175$ $\mathrm{GeV}, \sqrt{S}=1.8 \mathrm{TeV}$, and $\mu=m$. The full range of $\eta$ extends to 25 , but we display the behavior only in the near-threshold region where resummation is important. We observe that, at the energy of the Tevatron, resummation is significant for the $q \bar{q}$ channel and less so for the $g g$ channel. In Fig. 1, the dotted curve shows that our final resummed cross section in the $q \bar{q}$ channel, after integration over all $\eta$, lies about half-way between the cross sections obtained from the near-threshold leading logarithms at orders $\mathcal{O}\left(\alpha^{3}\right)$ and $\mathcal{O}\left(\alpha^{4}\right)$. The latter have been integrated over the region $0<z<1$.

We display our inclusive total production cross section as a function of the heavy quark

mass in Fig. 4. The central value of our predictions is defined as the value obtained with 
the choice $\mu / m=1$, and the lower and upper limits are the maximum and minimum of the cross section in the range of the hard scale $\mu / m \in\{0.5,2\}$. This definition of the central value is common, but it results here in an asymmetric uncertainty estimate; the extent of the range above the central value is smaller than that below. At $m=175 \mathrm{GeV}$, the full width of the uncertainty band is about $10 \%$. In Fig. 5, we show the variation of our resummed cross section as the value of the renormalization/factorization scale $\mu$ is changed. As is to be expected, less variation with $\mu$ is evident in the resummed cross section than in the next-to-leading order cross section, also shown in Fig. 5. We remark that the cross section reaches its maximum at a value of $\mu$ just slightly larger than $m / 2$. We consider that the variation of the cross section over the range $\mu / m \in\{0.5,2\}$ provides a good overall estimate of uncertainty. For comparison, we note that over the same range of $\mu$, the strong coupling strength $\alpha$ varies by $\pm 10 \%$ at $m=175 \mathrm{GeV}$. Using a different choice of parton densities [13], we find a $4 \%$ difference in the central value of our prediction [2] at $m=175 \mathrm{GeV}$. A comparison of the predictions [3] in the $\overline{\mathrm{MS}}$ and DIS factorization schemes also shows a modest difference at the level of $4 \%$.

In estimating uncertainties, we do not consider explicit variations of our non-perturbative boundary, expressed through Eq. (177). For a fixed $m$ and $\mu$, Eq. (17) is obtained by enforcing dominance of the leading hard kernel (determined through perturbative power-counting) over the subleading ones, all of which are calculable. Therefore, Eq. (17) is derived and is not a source of uncertainty. However, at fixed $m$, the boundary necessarily varies as $\mu$ and thus $\alpha$ vary.

Our prediction of the cross section in Fig. 4 is in agreement with the data on top quark production [7]. We find $\sigma^{t \bar{t}}(m=175 \mathrm{GeV}, \sqrt{S}=1.8 \mathrm{TeV})=5.52_{-0.42}^{+0.07} p b$. The central value of this cross section is larger than the next-to-leading order value at $\mu=m$ by about $9 \%$.

Extending our calculation at $\sqrt{S}=1.8 \mathrm{TeV}$ to much larger values of $m$ than shown in Fig. 4, we find that resummation in the principal $q \bar{q}$ channel produces enhancements over the next-to-leading order cross section of $21 \%, 26 \%$, and $34 \%$, respectively, at $m=500,600$, and $700 \mathrm{GeV}$. The reason for the increase of the enhancements with mass at fixed energy is that 
the threshold region becomes increasingly dominant. Since the $q \bar{q}$ channel also dominates in the production of hadronic jets at very large values of transverse momenta, we suggest that on the order of $20 \%$ of the excess cross section reported by the CDF collaboration [14] may well be accounted for by resummation.

The top quark cross section increases quickly with the energy of the $p \bar{p}$ collider. We provide predictions in Fig. 6 for an upgraded Tevatron operating at $\sqrt{S}=2$ TeV. We determine $\sigma^{t \bar{t}}(m=175 \mathrm{GeV}, \sqrt{S}=2 \mathrm{TeV})=7.56_{-0.55}^{+0.10} \mathrm{pb}$. The $2 \mathrm{pb}$ increase in the predicted top quark cross section over its value at $\sqrt{S}=1.8 \mathrm{TeV}$ is about a $37 \%$ gain. The central value rises to $22.4 \mathrm{pb}$ at $\sqrt{S}=3 \mathrm{TeV}$ and $46 \mathrm{pb}$ at $\sqrt{S}=4 \mathrm{TeV}$. For a fixed mass of the heavy quark, the fraction of the cross section supplied by the $g g$ subprocess increases rapidly. For $m=175 \mathrm{GeV}$, this fraction is about $15 \%$ at $\sqrt{S}=2 \mathrm{TeV}$ and $51 \%$ at $\sqrt{S}=4$ $\mathrm{TeV}$.

Turning to $p p$ scattering at the energies of the Large Hadron Collider (LHC) at CERN, we note a few significant differences from $p \bar{p}$ scattering at the energy of the Tevatron. The dominance of the $q \bar{q}$ production channel is replaced by $g g$ dominance at the LHC. Owing to the much larger value of $\sqrt{S}$, the near-threshold region in the subenergy variable is relatively less important, reducing the significance of initial-state soft gluon radiation. Lastly, physics in the region of large subenergy, where straightforward next-to-leading order QCD is also inadequate, becomes significant for $t \bar{t}$ production at LHC energies. Using the approach described in this paper, we estimate $\sigma^{t \bar{t}}(m=175 \mathrm{GeV}, \sqrt{S}=14 \mathrm{TeV})=760 \mathrm{pb}$.

\section{OTHER METHODS OF RESUMMATION}

The groups of Laenen, Smith, and van Neerven (LSvN) and of Catani, Mangano, Nason, and Trentadue (CMNT) have also published predictions for the total cross section based on resummation of initial state soft gluon radiation. At $m=175 \mathrm{GeV}$ and $\sqrt{S}=1.8 \mathrm{TeV}$, the three values are: $\left.\sigma^{t \bar{t}}(\mathrm{BC}[2] .3]\right)=5.52_{-0.42}^{+0.07} \mathrm{pb} ; \sigma^{t \bar{t}}(\mathrm{LSvN}$ 迎 $)=4.95_{-0.40}^{+0.70} \mathrm{pb}$; and

$\sigma^{t \bar{t}}(\mathrm{CMNT}$ 顿 $)=4.75_{-0.68}^{+0.63} \mathrm{pb}$. From the purely numerical point of view, all three pre- 
dictions agree within their estimates of theoretical uncertainty. However, the resummation methods differ, the methods for estimating the uncertainties differ, and different parton sets are used. Comparing with LSvN [1], we find that our central values are $10-14 \%$ larger, and our estimated theoretical uncertainty is $9-10 \%$ compared with their $28 \%-20 \%$. The larger central value is attributable, in part, to the use of different parton densities; our Born cross section is about $3-5 \%$ larger than the LSvN Born cross section. However, it is the choice of the infrared cutoff $\mu_{o}$ in the LSvN method that controls the size of their cross section. The cutoff $\mu_{o}$ is selected so that the resummed cross section is about equal to the next-to-nextto-leading order leading-logarithm cross section $\sigma_{i j}^{(0+1+2)}$, obtained from Eq. (5). In contrast, in our approach the non-perturbative boundary $z_{\max }$ is derived within the context of the calculation by the requirement that the universal leading-logarithmic terms be dominant. There is no a priori reason that our resummed result should be only $10 \%$ greater than the next-to-leading order cross section at $m=175 \mathrm{GeV}$ and $\sqrt{S}=1.8 \mathrm{TeV}$. As such, we regard the approximate agreement of our result and that of LSvN as somewhat fortuitous. Both the central value and the band of uncertainty of the LSvN predictions are sensitive to their infrared cutoffs, as we described previously [3].

From a theoretical point of view, study of the variation of the predicted cross section with the hard scale $\mu$, illustrated here in Fig. 5 , is important because it reflects the stability of the calculation under changes of a perturbative but not directly determinable renormalizationfactorization scale. One of the advantages of a resummation calculation should be diminished dependence of the cross section on $\mu$, less variation than is present in a fixed-order calculation. To estimate theoretical uncertainty, we use the standard $\mu$ variation, and we find a band of uncertainty of about $10 \%$ at $m=175 \mathrm{GeV}$ and $\sqrt{S}=1.8 \mathrm{TeV}$. The LSvN group obtain their uncertainty primarily from variations of their infrared cutoff whose role is to measure ignorance of non-perturbative effects in that approach.

The group of Catani, Mangano, Nason, and Trentadue (CMNT) [⿴囗 calculate a central value of the resummed cross section (also with $\mu / m=1$ ) that is less than $1 \%$ above the exact next-to-leading order value. There are similarities and differences between our approach to 
resummation and the method of Ref. [4]. We both begin in moment space with the same universal leading-logarithm expression, but differences occur after the transformation to momentum space. In this paper, we set aside comments on mathematical aspects of their procedure and focus instead on phenomenological issues of interest. As remarked above, the Mellin transformation generates subleading terms in momentum space. The suppression of the effects of resummation arises from the retention in Ref. [四] of numerically significant non-universal subleading logarithmic terms.

CMNT choose to retain all of these inasmuch as they perform the Mellin inversion numerically. Instead, in keeping with the fact that subleading logarithmic terms are not universal, we retain only the universal leading logarithm terms in momentum space, and we restrict our phase space integration to the region in which the subleading terms would not be numerically significant regardless. The differences in the two approaches can be stated more explicitly if we examine the perturbative expansion of the kernel $\mathcal{H}_{i j}^{R}(z, \alpha)$, Eq. (15). If, instead of restricting the resummation to the universal leading logarithms only, we were to use the full content of Eq. (15), we would arrive at an analytic expression that is equivalent to the numerical inversion of Ref. [4],

$$
\mathcal{H}_{i j}^{R} \simeq 1+2 \alpha C_{i j}\left[\ln ^{2}(1-z)+2 \gamma_{E} \ln (1-z)\right]+\mathcal{O}\left(\alpha^{2}\right)
$$

where $\gamma_{E}$ is Euler's constant. In terms of this expansion, in our work we retain only the leading term $\ln ^{2}(1-z)$ at order $\alpha$, but CMNT retain both this term and the subleading term $2 \gamma_{E} \ln (1-z)$. Indeed, if the subleading term $2 \gamma_{E} \ln (1-z)$ is discarded in Eq. (19), the residuals $\delta_{i j} / \sigma_{i j}^{N L O}$ defined in Ref. [4] increase from $0.18 \%$ to $1.3 \%$ in the $q \bar{q}$ production channel and from $5.4 \%$ to $20.2 \%$ in the $g g$ channel [15]. After addition of the two channels, the total residual $\delta / \sigma^{N L O}$ grows from the negligible value of about $0.8 \%$ cited in Ref. [4] to the value $3.5 \%$. While still smaller than the increase of about $9 \%$ that we obtain, the increase of $3.5 \%$ vs. $0.8 \%$ shows the substantial influence of the subleading logarithmic terms retained in Ref. [4].

We judge that it is preferable to integrate over only the region of phase space in which the 
subleading term is suppressed numerically. Our reasons include the fact that the subleading term is not universal, is not the same as the subleading term in the exact $\mathcal{O}\left(\alpha^{3}\right)$ calculation, and can be changed if one elects to keep non-leading terms in moment space. The subleading term is negative and numerically very significant when it is integrated throughout phase space (i.e., into the region of $z$ above our $z_{\max }$ ). In the $q \bar{q}$ channel at $m=175 \mathrm{GeV}$ and $\sqrt{S}=1.8 \mathrm{TeV}$, its inclusion eliminates more than half of the contribution from the leading term. In our view, the presence of numerically significant subleading contributions begs the question of consistency. A further justification for the retention of only the leading term is that it approximates the exact next-to-leading order result well, as shown in Fig. 1. The choice made in Ref. [4] reproduces only one-third of the exact next-to-leading order

result. The influence of subleading terms is amplified at higher orders where additional subleading structures occur in the approach of Ref. [4] with significant numerical coefficients proportional to $\pi^{2}, \zeta(3)$, and so forth. We present a more detailed discussion of this issue in the next section.

\section{FURTHER DISCUSSION OF THE CMNT APPROACH}

In this section we offer a more systematic analysis of the role played in the approach of Ref. [4 by non-universal subleading logarithms. We are interested in expansions of the resummed momentum-space kernel, Eq. (15), up to two loops. Therefore, the corresponding cross sections are integrable down to threshold, $z_{\max }=1$ and $\eta=0$. As we will see, though, the effects of the various classes of logarithms are pronounced if one continues the region of integration outside our perturbative regime.

In moment space, the exponent to two-loops is obtained from Eq. (11):

$$
E_{i j}^{[2]}(x, \alpha)=g \alpha\left(s_{2,1} x^{2}+s_{1,1} x+s_{0,1}\right)+g \alpha^{2}\left(s_{3,2} x^{3}+s_{2,2} x^{2}+s_{1,2} x+s_{0,2}\right),
$$

with $g=2 C_{i j}$ and $x=\ln n$. The corresponding hard kernels in momentum space can be derived from Eq. (15), according to the formulas (91) through (94) of Ref. [3]. In the 
notation of Ref. [3], we retain terms up to those that are linear in $P_{2}$. Alternatively, one can perform the analytical Mellin inversion directly, beginning with Eq. (20). The two methods provide identical results down to the monomials $x_{z}^{2} \alpha^{2} ; x_{z} \equiv \ln (1 /(1-z))$. Here we quote results based on the explicit inversion of Eq. (20). (The monomial $x_{z} \alpha^{2}$ can be obtained also in the first approach if we keep the quadratic term in $P_{2}$.) After a trivial integration is performed, the results for the one- and two-loop hard kernels are

$$
\mathcal{H}^{(1)}=x_{z}^{2} \alpha\left\{g s_{2,1}\right\}+x_{z} \alpha\left\{g\left(s_{1,1}+2 c_{1} s_{2,1}\right)\right\}
$$

and

$$
\begin{aligned}
& \mathcal{H}^{(2)}=x_{z}^{4} \alpha^{2}\left\{g^{2} s_{2,1}^{2} / 2\right\}+x_{z}^{3} \alpha^{2}\left\{g s_{3,2}+g^{2}\left(s_{2,1} s_{1,1}+2 c_{1} s_{2,1}^{2}\right)\right\} \\
& +x_{z}^{2} \alpha^{2}\left\{g\left(s_{2,2}+3 c_{1} s_{3,2}\right)+g^{2}\left(s_{1,1}^{2} / 2+3 c_{1} s_{1,1} s_{2,1}+s_{2,1} s_{0,1}+s_{2,1}^{2}\left[6 c_{2}-\pi^{2}\right]\right\}\right. \\
& +x_{z} \alpha^{2}\left\{g\left(s_{1,2}+2 c_{1} s_{2,2}+s_{3,2}\left[6 c_{2}-\pi^{2}\right]\right)\right. \\
& \left.+g^{2}\left(s_{0,1} s_{1,1}+2 c_{1} s_{0,1} s_{2,1}+c_{1} s_{1,1}^{2}+s_{2,1} s_{1,1}\left[6 c_{2}-\pi^{2}\right]+s_{2,1}^{2}\left[12 c_{3}-2 \pi^{2} c_{1}\right]\right)\right\} .
\end{aligned}
$$

All the constants are defined in Eqs. (11) and (12). We remark that Eq. (21) includes a leading logarithmic term, $x_{z}^{2} \alpha$, as well as a next-to-leading term, $x_{z} \alpha$.

The question we now address is whether it is justified and meaningful to retain all of the terms in Eqs. (21) and (22) in the computation of the resummed cross section. The issue has to do with what one intends by resummation of leading logarithms. We use the term leading logarithm resummation to denote the case in which the moment space exponent, Eq. (20), contains only the constants $E_{L L}=\left\{s_{\rho+1, \rho}, 0\right\}$. This is also what is done in the method of Ref. 迎, and the exponent in moment space in their work is identical to that used for our predictions, Eq. (14). However, in contrast to our expression in momentum space, Eq. (16), the corresponding expression in momentum space of Ref. [4] includes the numerical equivalent of all terms in Eqs. (21) and (22) that are proportional to $s_{\rho+1, \rho}$.

If expressed analytically, the corresponding "LL" hard kernels in the method of Ref. [4] are

$$
\mathcal{H}_{L L}^{(1)}=x_{z}^{2} \alpha g-x_{z} \alpha 2 g \gamma_{E}
$$


and

$$
\begin{aligned}
& \mathcal{H}_{L L}^{(2)}=x_{z}^{4} \alpha^{2} g^{2} / 2+x_{z}^{3} \alpha^{2}\left\{2 g b_{2} / 3-2 \gamma_{E} g^{2}\right\} \\
& +x_{z}^{2} \alpha^{2}\left\{-2 g b_{2} \gamma_{E}+g^{2}\left[3 \gamma_{E}^{2}-\pi^{2} / 2\right]\right\} \\
& +x_{z} \alpha^{2}\left\{2 g b_{2}\left[3 \gamma_{E}^{2}-\pi^{2} / 2\right] / 3+g^{2}\left[\gamma_{E} \pi^{2}-2 \gamma^{3}-4 \zeta(3)\right]\right\},
\end{aligned}
$$

where $\zeta(s)$ is the Riemann zeta function; $\zeta(3)=1.2020569$. Evaluating the expressions numerically for the $q \bar{q}$ channel, we obtain

$$
\mathcal{H}_{L L}^{(1)}=x_{z}^{2} \alpha \times 2.66666-x_{z} \alpha \times 3.07848
$$

and

$$
\mathcal{H}_{L L}^{(2)}=x_{z}^{4} \alpha^{2} \times 3.55555-x_{z}^{3} \alpha^{2} \times 4.80189-x_{z}^{2} \alpha^{2} \times 33.88456-x_{z} \alpha^{2} \times 9.82479 .
$$

Apart from the leading monomials that are the same as those in our approach, Eqs. (25) and (26) include a series of subleading terms, each of which has a significant negative coefficient. In practice, these subleading terms in the approach of Ref. [4 suppress the effects of resummation essentially completely. One of the effects of this suppression is that the resummed partonic cross section is smaller than its next-to-leading order counterpart in the neighborhood of $\eta=0.1$, a region in which the next-to-leading order partonic cross section takes on its largest values. This point is illustrated in Fig. 3 of the second paper in Ref. [4].

Although the specific set of subleading terms in Eqs. (25) and (26) is generated in the inversion of the Mellin transform, we would argue that the terms are accidental, at best. Our reasoning is based on an examination of the exact next-to-leading order calculation of the cross section for heavy quark production and of similar calculations of the Drell-Yan process up to two-loops. First, terms involving $\gamma_{E}$ do not appear in the exact next-to-leading order calculation of the hard part, since they are removed in the specification of the $\overline{\mathrm{MS}}$ factorization scheme. Therefore, the term proportional to $\gamma_{E}$ in Eq. (23) is suspect. Second, if we extract the specific value of the subleading logarithm from the full $\mathcal{O}\left(\alpha^{3}\right)$ next-toleading order calculation [5], we find [16] $x_{z} \alpha(2 g-41 / 6)$ instead of the term $-x_{z} \alpha 2 g \gamma_{E}$ in the 
equivalent CMNT Eq. (23). Referring to Eq. (25), we remark that instead of the numerical coefficient 3.07848 , one would have the smaller value 1.5 if the subleading logarithm of the exact $\mathcal{O}\left(\alpha^{3}\right)$ calculation were used. Thus, not only is the $\mathcal{O}(\alpha)$ subleading term retained in the approach of Ref. [4] different from that of the exact calculation, it is numerically about twice as large. Third, we would claim that the results of a LL resummation should not rely on the subleading structures in any significant way. However, in the approach of Ref. 沺, Eq. (23), which is the one-loop projection of their resummed prediction, reproduces only $1 / 3$ of the exact $\mathcal{O}\left(\alpha^{3}\right)$ enhancement, the other $2 / 3$ being cancelled by the second (non-universal) term of Eq. (23). Correspondingly, the method of Ref. 四 fails an important consistency check: it sets out to resum the threshold corrections responsible for the large enhancement of the cross section at next-to-leading order; in the end, it does not reproduce most of this enhancement.

Addressing questions associated with the $\gamma_{E}$ terms [17] CMNT examine a type of NLL resummation in the second paper of Ref. 四. In this NNL resummation, the $\left\{s_{\rho+1, \rho}, s_{\rho, \rho}\right\}$ terms are retained in the exponent of Eq. (201). The corresponding hard kernels become

$$
\mathcal{H}_{N L L}^{(1)}=x_{z}^{2} \alpha g
$$

and

$$
\mathcal{H}_{N L L}^{(2)}=x_{z}^{4} \alpha^{2} g^{2} / 2+x_{z}^{3} \alpha^{2} 2 g b_{2} / 3-x_{z}^{2} \alpha^{2} g^{2}\left[\gamma_{E}^{2}+\pi^{2} / 2\right]-x_{z} \alpha^{2}\left\{g b_{2}\left[2 \gamma_{E}^{2}+\pi^{2} / 3\right]+g^{2} 4 \zeta(3)\right\} .
$$

Comparing Eqs. (27) and (28) with Eq. (5), we observe that Eq. (27) is identical to the oneloop projection of our hard kernel. As shown in Ref. [3], it yields an excellent approximation to the exact next-to-leading order cross section. On the other hand, our two-loop projection contains only the first two terms of Eq. (28). The term proportional to $x_{z}^{3} \alpha^{2}$ is present in our case, along with the leading term proportional to $x_{z}^{4} \alpha^{2}$, because it comes from the leading logarithms in the exponent $E(n)$, through one-loop running of the coupling strength. In contrast to Eq. (24), Eq. (28) relegates the influence of the ambiguous constant coefficients 
to lower powers of $x_{z}$ (but with larger negative coefficients). In the amended scheme of Ref. [4], the unphysical $\gamma_{E}$ terms are still present in the two-loop result, Eq. (28), along with $\pi^{2}$ and $\zeta(3)$ terms that may be expected but whose coefficients have no well defined physical origin. Recast in numerical form, Eqs. (27) and (28) become

$$
\mathcal{H}_{N L L}^{(1)}=x_{z}^{2} \alpha \times 2.66666
$$

and

$$
\mathcal{H}_{N L L}^{(2)}=x_{z}^{4} \alpha^{2} \times 3.55555+x_{z}^{3} \alpha^{2} \times 3.40739-x_{z}^{2} \alpha^{2} \times 37.46119-x_{z} \alpha^{2} \times 54.41253
$$

We call attention to the significant difference between the coefficients of all but the very leading power of $x_{z}$ in Eqs. (25) and (26) with respect to those in Eqs. (29) and (30), and to the fact that the numerical coefficients grow in magnitude as the power of $x_{z}$ decreases.

Using their NLL amendment, CMNT find that the central value of their resummed cross section exceeds the next-to-leading order result by $3.5 \%$ (both $q \bar{q}$ and $g g$ channels added). This increase is about 4 times larger than the central value of the increase obtained in their first method, closer to our increase of about $9 \%$. The reason for the significant change of the increase resides with the subleading structures, viz., in the differences between the LL version Eqs. (25) and (26) and the NLL version Eqs. (29) and (30). The subleading terms at two-loops cause a total suppression of the two-loop contribution (in fact, that contribution is negative), if one integrates all the way into what we call the non-perturbative regime. This suppression explains why an enhancement of only $3.5 \%$ is obtained in the amended method of Ref. [4], rather than our $9 \%$.

CMNT argue that retention of their subleading terms in momentum space is important for "energy conservation". By this statement, they mean that one begins the formulation of resummation with an expression in momentum space containing a delta function representing conservation of the fractional partonic momenta. In moment space, this delta function subsequently unconvolves the resummation. Therefore, when one inverts the Mellin transform to return to momentum space, the full set of logarithms generated by this inversion are 
required by the original energy conservation. This line of reasoning would be compelling if the complete exponent $E(n)$ in moment space were known exactly, i.e., if the resummation in moment space were exact in representing the cross section to all orders. However, the exponent is truncated in all approaches, and knowledge of the logarithms it resums reliably is limited both in moment and in momentum space. Hence, the set of logarithms produced by the Mellin inversion in momentum space should also be restricted. In our approach energy conservation is obeyed in momentum space consistently with the class of logarithms resummed. On the other hand, in the method of Ref. 近, knowledge is claimed of all logarithms generated from the Mellin inversion, despite the fact that the truncation in moment space makes energy conservation a constraint restricted to the class of logarithms that is resummable, i.e., a constraint restricted by the truncation of the exponent $E(n)$. The two approaches would be equivalent provided a constraint be in place on the effects of subleading logarithms. This constraint is precisely our Eq. (17). By contrast, no such constraint is furnished in Ref. [4]. For this reason the results of Ref. [4] are numerically unstable if one set of the logarithms generated in momentum space is adopted as "the set corresponding to energy conservation", and then compared with another set, produced by a different truncation of $E(n)$.

We have identified the terms responsible for the difference between our answer for the resummed cross section and that of Ref. [4. These differences reside with subleading logarithms whose presence is not substantiated by physical arguments. The essence of our determination of the perturbative regime in Eq. (17) is precisely that, in this regime, subleading structures are also numerically subleading, whether or not the classes of subleading logarithms coming from different truncation of the master formula for the resummed hard kernel, Eq. (15), are included. The results presented in Fig. 11 of Ref. [3], show that if we alter our resummed hard kernel to account for subleading structures but still stay within our perturbative regime, the resulting cross section is reduced by about $4 \%$, within our band of perturbative uncertainty.

A criticism [4] is that of putative "spurious factorial growth" of our resummed cross 
section, above and beyond the infrared renormalons that are eliminated from our approach. The issue, as we demonstrated in Eq. (29) of Ref. [3], can be addressed most easily if we substitute any monomial appearing in Eq. (22), symbolically $\alpha^{m} c(l, m) \ln ^{l} x_{z}$, into Eq. (3) and integrate over $z$ :

$$
\alpha^{m} c(l, m) \int_{z_{\min }}^{1} d z \ln ^{l} x_{z}=\alpha^{m} c(l, m)\left(1-z_{\min }\right) l ! \sum_{j=0}^{l} \ln ^{j}\left(1 /\left(1-z_{\text {min }}\right)\right) .
$$

For the purposes of this demonstration we set $\hat{\sigma}_{i j}^{\prime}=1$. The coefficients $c(l, m)$ can be read directly from Eq. (22). For the leading logarithmic terms,

$$
c(2 m, m) \propto 1 / m !
$$

where this factorial comes directly from exponentiation. After the integration over the entire $z$-range, the power of the logarithm in $x_{z}$ becomes a factorial multiplicative factor, $l$ !. The presence of $l$ ! follows directly from the existence of the powers of $\ln x_{z}$ that are present explicitly in the finite-order result in $\mathrm{pQCD}$ and is therefore inevitable. If this exercise is repeated, but with the range of integration in Eq. (31) constrained to our perturbative regime, one obtains the difference between the right-hand-side of Eq. (31) and a similar expression containing $z_{\max }$. The result is numerically smaller, but both of the pieces are multiplied by $l !$.

The factorial coefficient $l$ ! is neither the only nor the most important source of enhancement. For the leading logarithms at two-loop order, $l=2 m=4$, and the overall combinatorial coefficient from Eqs. (31) and (32) is $(2 m) ! / m !=12$. For comparison, at representative values of $\eta$ near threshold, $\eta=0.1$ and 0.01 , the sum of logarithmic terms in Eq. (31) provides factors of 16.1 and 314.3, respectively. Similarly, the (multiplicative) color factors at this order of perturbation theory are $\left(2 C_{i j}\right)^{2}=7.1$ and 36 for the $q \bar{q}$ and gg channels, respectively. All of these features are connected to the way threshold logarithmic contributions appear in finite-order pQCD and how they signal the presence of the non-perturbative regime. Thus, preoccupation with the $l$ ! factor seems misplaced.

The phrase "spurious factorial growth" appears to rename the logarithmic enhancements present in Eqs. (21) and (22), after the integral over $z$. On the other hand, according to our 
understanding, the claim [4] of "absence of factorial growth" is based on the use in Ref. [4] of Eq. (26) for their main predictions, an expression that contains non-universal subleading logarithms, all with significant negative coefficients. Mathematically, factorial growth is present for each of the powers of the logarithm in Eq. (31), since these monomials are irreducible (linearly independent). Absence of factorial growth based on a numerical cancellation between various classes of logarithms, most of them with physically unsubstantiated coefficients, appears to us to be an incorrect use of terminology, rather than a transparent expression of the mathematics.

From a purely phenomenological point of, one cannot claim that a $9 \%$ increase of the top quark cross section at $m=175 \mathrm{GeV}$ and $\sqrt{S}=1.8 \mathrm{TeV}$ reveals factorial growth but that an $0.8 \%$ increase does not. In the approach taken in Ref. [4], the effects of resummation are suppressed by a series of subleading logarithms with large negative coefficients. If there is no physical basis for preference of Eqs. (23) and (24) over Eqs. (27) and (28), as the authors of Ref. [4] seem to suggest, then the difference in the resulting cross sections can be interpreted as a measure of theoretical uncertainty. This interpretation would not justify firm conclusions of a minimal $0.8 \%$ increment based on the choice of Eqs. (23) and (24).

As remarked in Sec. IV, the value quoted in Ref. [4] for the physical cross section at $m=$ $175 \mathrm{GeV}$ and $\sqrt{S}=1.8 \mathrm{TeV}$, including theoretical uncertainty, lies within our uncertainty band. Therefore, the numerical differences between us for the specific case of top quark production at the Tevatron have little practical significance. However, there are important differences of principal in our treatment of subleading contributions that will have more significant consequences for predictions in other processes or at other values of top mass and/or at other energies, particularly in reactions dominated by $g g$ subprocesses.

\section{DISCUSSION AND CONCLUSIONS}

In this paper, we present and discuss the calculation of the inclusive cross section for top quark production in perturbative QCD, including the resummation of initial-state gluon 
radiation to all orders in the strong coupling strength. The advantages of the perturbative resummation method [2.:3] we espouse are that there are no arbitrary infrared cutoffs and there is a well-defined perturbative region of applicability where subleading logarithmic terms are numerically suppressed. Our theoretical analysis shows that perturbative resummation without a model for non-perturbative behavior is both possible and advantageous. In perturbative resummation, the perturbative region of phase space is separated cleanly from the region of non-perturbative behavior.

When evaluated for top quark production at $\sqrt{S}=1.8 \mathrm{TeV}$, our resummed cross sections are about $9 \%$ above the next-to-leading order cross sections computed with the same parton distributions. The renormalization/factorization scale dependence of our cross section is fairly flat, resulting in a $9-10 \%$ theoretical uncertainty. This variation is smaller than the corresponding dependence of the next-to-leading cross section, as should be expected. Our perturbative boundary of $1.22 \mathrm{GeV}$ above the threshold in the dominant $q \bar{q}$ channel is comparable to the hadronic width of the top quark, a natural definition of the perturbative boundary. Neither this, nor the somewhat larger value of $8.64 \mathrm{GeV}$ above threshold in the $g g$ channel, associated with the larger color factor in the $g g$ channel, is "unphysically large" [4]. In recent papers [4], the authors state that the increase in cross section they find with their resummation method is no more than $1 \%$ over next-to-leading order. The numerical difference in the two approaches boils down to the treatment of the subleading logarithms, which can easily shift the results by a few percent, if proper care is not taken. Our approach includes the universal leading logarithms only while theirs includes non-universal subleading structures which produce the suppression they find. In Sec. VI, we explain why we judge that that our treatment of the subleading structures is preferable.

Our theoretical analysis and the stability of our cross sections under $\mu$ variation provide confidence that our perturbative resummation procedure yields an accurate calculation of the inclusive top quark cross section at Tevatron energies and exhausts present understanding of the perturbative content of the theory. Our resummed top quark cross section is about $9 \%$ above the next-to-leading order cross section with an estimated theoretical uncertainty of 
$9-10 \%$, associated with $\mu$ variation. An entirely different procedure to estimate the overall theoretical uncertainty is to compare our enhancement of the cross section above the nextto-leading order value to that of Ref. [4], again yielding about 10\%. An interesting question is whether theory can aspire to an accuracy of better than $10 \%$ for the calculation of the top quark cross section. To this end, a more complete mastery of subleading logarithms would be desirable, perhaps requiring a formidable complete calculation at next-to-next-to-leading order of heavy quark production, to establish the possible pattern of subleading logarithms, and resummation of both leading and subleading logarithms [18].

Our prediction agrees with data, within the large experimental uncertainties. Despite the different treatment of subleading terms, our calculation of the inclusive cross section for top quark production at the Fermilab Tevatron and that of Ref. [4 fall within the estimated uncertainties of each other. If a cross section significantly different from ours is measured in future experiments at the Tevatron with greater statistical precision, we would look for explanations in effects beyond QCD perturbation theory. These explanations might include unexpectedly substantial non-perturbative effects or new production mechanisms. An examination of the distribution in $\eta$ might be revealing.

In this paper, we concentrate on the all-orders summation of large logarithmic terms that are important in the near-threshold region of small values of the scaled partonic subenergy, $\eta \rightarrow 0$. Our specific case is top quark production at the Fermilab Tevatron collider. Other processes for which threshold resummation and our methodology will also be pertinent include the production of hadronic jets that carry large values of transverse momentum and the production of pairs of supersymmetric particles with large mass. There is a complementary region of large $\eta, \eta \rightarrow 1$, in which the resummation of different large logarithms may also be important. The production of heavy quarks $Q$ in the limit that the hadronic center-of-mass energy is much larger than the quark mass provides an example. The dominant production channel is $g g \rightarrow Q \bar{Q} X$; the ratio of the next-to-leading order partonic cross section divided by its leading-order approximation is very large at large $\eta$. Correspondingly, the fixed-order cross section will not offer a reliable prediction, and an all-orders approach is 
called for [19]. Particular cases include the total cross sections for bottom quark production at the Tevatron and top quark production at the CERN Large Hadron Collider.

\section{ACKNOWLEDGMENTS}

Work in the High Energy Physics Division at Argonne National Laboratory is supported by the U.S. Department of Energy, Division of High Energy Physics, Contract W-31-109ENG-38. ELB is grateful to J. Schlereth for valuable and timely assistance. 


\section{REFERENCES}

[1] E. Laenen, J. Smith, and W.L. van Neerven, Nucl. Phys. B369, 543 (1992); Phys. Lett. B 321, 254 (1994).

[2] E. L. Berger and H. Contopanagos, Phys. Lett. B 361, 115 (1995).

[3] E. L. Berger and H. Contopanagos, Phys. Rev. D 54, 3085 (1996).

[4] S. Catani, M. Mangano, P. Nason, and L. Trentadue, Phys. Lett. B 378, 329 (1996); Nucl. Phys. B478, 273 (1996). S. Catani, hep-ph/9610413, to be published in the Proceedings of QCD Euroconference 96, Montpellier, France, July, 1996. S. Frixione, M. L. Mangano, P. Nason, and G. Ridolfi, hep-ph/9702287 (Feb., 1997).

[5] P. Nason, S. Dawson, and R.K. Ellis, Nucl. Phys. B303, 607 (1988); B327, 49 (1989); B335, 260(E) (1990). W. Beenakker, H. Kuijf, W.L. van Neerven, and J. Smith, Phys. Rev. D 40, 54 (1989); W. Beenakker, W.L. van Neerven, R. Meng, G.A. Schuler, and J. Smith, Nucl. Phys. B351, 507 (1991).

[6] S. Willenbrock and D. Dicus, Phys. Rev. D 34, 155 (1986); C.-P. Yuan, Phys. Rev. D 41, 42 (1990); R. K. Ellis and S. Parke, Phys. Rev. D 46, 3785 (1992); S. Cortese and R. Petronzio Phys. Lett. B 253, 494 (1991); T. Stelzer and S. Willenbrock, Phys. Lett. B 357, 125 (1995); M. Smith and S. Willenbrock, Phys. Rev. D 54, 6696 (1996).

[7] P. Tipton, Proceedings of the 28th International Conference on High Energy Physics, Warsaw, July, 1996, ed. by Z. Ajduk and A. K. Wroblewski (World Scientific, Singapore, 1997), 123; A. Castro (CDF collaboration), ibid, 851; S. Abachi (D0 collaboration), hepex/9703008 (Mar., 1997), submitted to Phys. Rev. Lett., hep-ex/9704015 (April, 1997), submitted to Phys. Rev. Lett..

[8] One might expect intuitively that the emission of soft gluons would suppress the cross section, not enhance it. The physical cross section is expressed through the factorization theorem, Eq. (1). Part of the effect of the gluonic radiative corrections is included in 
the parton densities. The remainder is a positive enhancement in the partonic cross sections. For a general discussion of the reason for the positive signs, see N. Kidonakis and G. Sterman in Les Rencontres de Physique de la Vallee d'Aoste, LaThuile, Aosta Valley, March, 1996, ed. by M. Greco (Frascati, 1996), 333 (hep-ph/9607222).

[9] H. Contopanagos and G. Sterman, Nucl. Phys. B400, 211 (1993); B419, 77 (1994). L. Alvero and H. Contopanagos, Nucl. Phys. B436, 184 (1995); B456, 497 (1995).

[10] G. Sterman, Nucl. Phys. B281, 310 (1987); S. Catani and L. Trentadue, Nucl. Phys. B327, 323 (1989); B353, 183 (1991); H. Contopanagos, E. Laenen, and G. Sterman, Nucl. Phys. B484, 303 (1997).

[11] Since the width of the top quark provides a physical definition of the perturbative boundary, it would not seem justified to integrate into the part of phase space that is above the value of $z\left(z_{\max }\right)$ and below the value of $\eta$ that correspond to this natural limit, as is done in Ref. [1].

[12] H.L. Lai et al., Phys. Rev. D51, 4763 (1995).

[13] A. Martin, R. Roberts, and W. J. Stirling, Phys. Lett. B354, 155 (1995).

[14] F. Abe et al. (CDF Collaboration), Phys. Rev. Lett. 77, 438 (1996).

[15] We thank Michelangelo Mangano and Paolo Nason for supplying the residuals for their gg channel that are not included in Ref. [4].

[16] The essence of this derivation may be found in Eqs.(122), (129), and (130) of Berger and Contopanagos, Ref. [3].

[17] This modification was considered after discussions with one of us (H.C.) during a visit to CERN in 1996.

[18] An analysis in moment space of the issues involved in resummation of subleading logarithms for heavy quark production is presented in N. Kidonakis and G. Sterman, Stony 
Brook report ITP-SB-97-24 (hep-ph/9705234), May, 1997.

[19] J. Collins and R. K. Ellis, Nucl. Phys. B360, 3 (1991). 


\section{FIGURES}

FIG. 1. Physical cross sections in the $q \bar{q}$ channel as a function of the heavy quark mass, in the $\overline{\mathrm{MS}}$ scheme. The solid lines denote the finite-order partial sums of the universal leading-logarithmic contributions from the explicit $\mathcal{O}\left(\alpha^{3}\right)$ and $\mathcal{O}\left(\alpha^{4}\right)$ calculations for the $t \bar{t}$ and Drell-Yan processes, respectively. Lower solid: $\sigma^{(0)}$; middle solid: $\sigma^{(0+1)}$; upper solid: $\sigma^{(0+1+2)}$. The dashed curve represents the exact next-to-leading order calculation for $t \bar{t}$ production, in excellent agreement with $\sigma^{(0+1)}$. The dotted curve is our resummed prediction.

FIG. 2. Optimum number of perturbative terms in the exponent with PVR. The solid family is for PVR and the dashed set for the perturbative approximation, both families increasing, for parametric values $n=10,20,30,40$.

FIG. 3. Differential cross sections $d \sigma / d \eta$ for $p \bar{p} \rightarrow t \bar{t} X$ at $\sqrt{S}=1.8 \mathrm{TeV}$ and $m=175 \mathrm{GeV}$ in the $\overline{\mathrm{MS}}$-scheme for (a) the $q \bar{q}$ and (b) the $g g$ channel: Born (dotted), next-to-leading order (dashed), and resummed (solid).

FIG. 4. Inclusive cross section for heavy quark production at $\sqrt{S}=1.8 \mathrm{TeV}$ in the $\overline{\mathrm{MS}}$ scheme. The dashed curves show our perturbative uncertainty band, while the solid curve is our central prediction.

FIG. 5. Renormalization/factorization hard scale dependence of the resummed (solid) and next-to-leading order (dashed) cross sections at $\sqrt{S}=1.8 \mathrm{TeV}$ for $m=175 \mathrm{GeV}$.

FIG. 6. Inclusive cross section for heavy quark production at $\sqrt{S}=2 \mathrm{TeV}$ in the $\overline{\mathrm{MS}}$ scheme. The dashed curves show our perturbative uncertainty band, while the solid curve is our central prediction. 


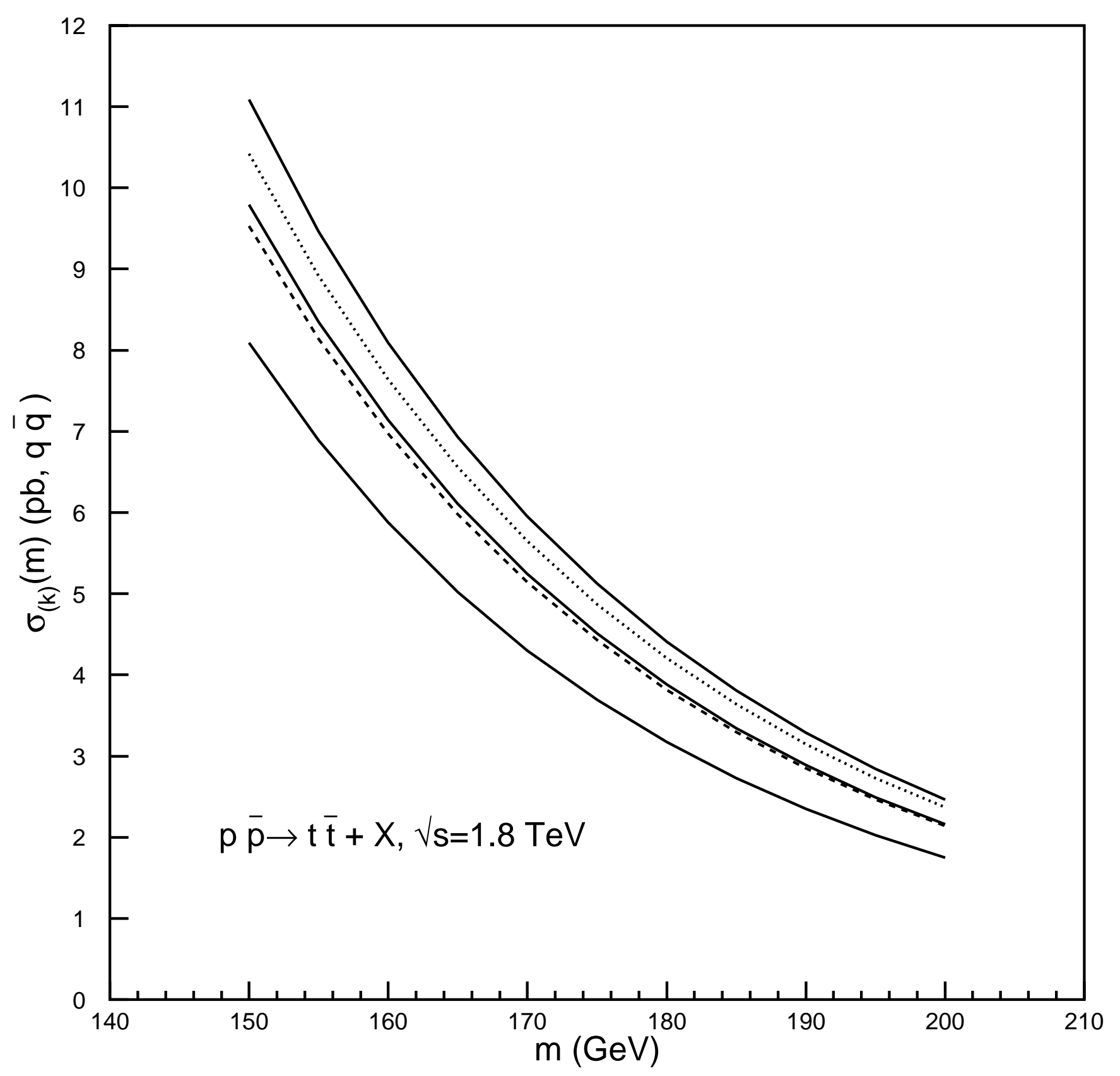

Figure 1 


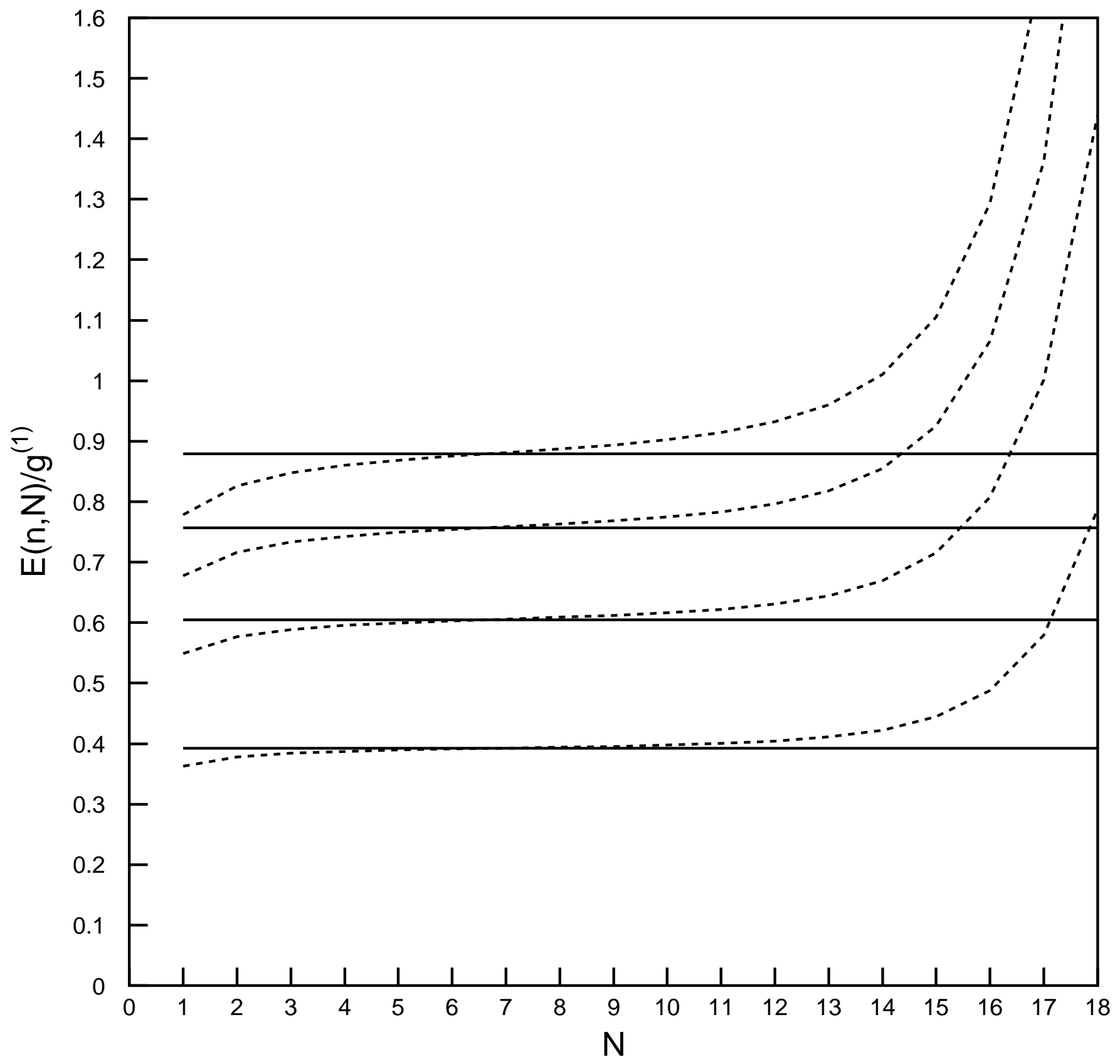

Figure 2 


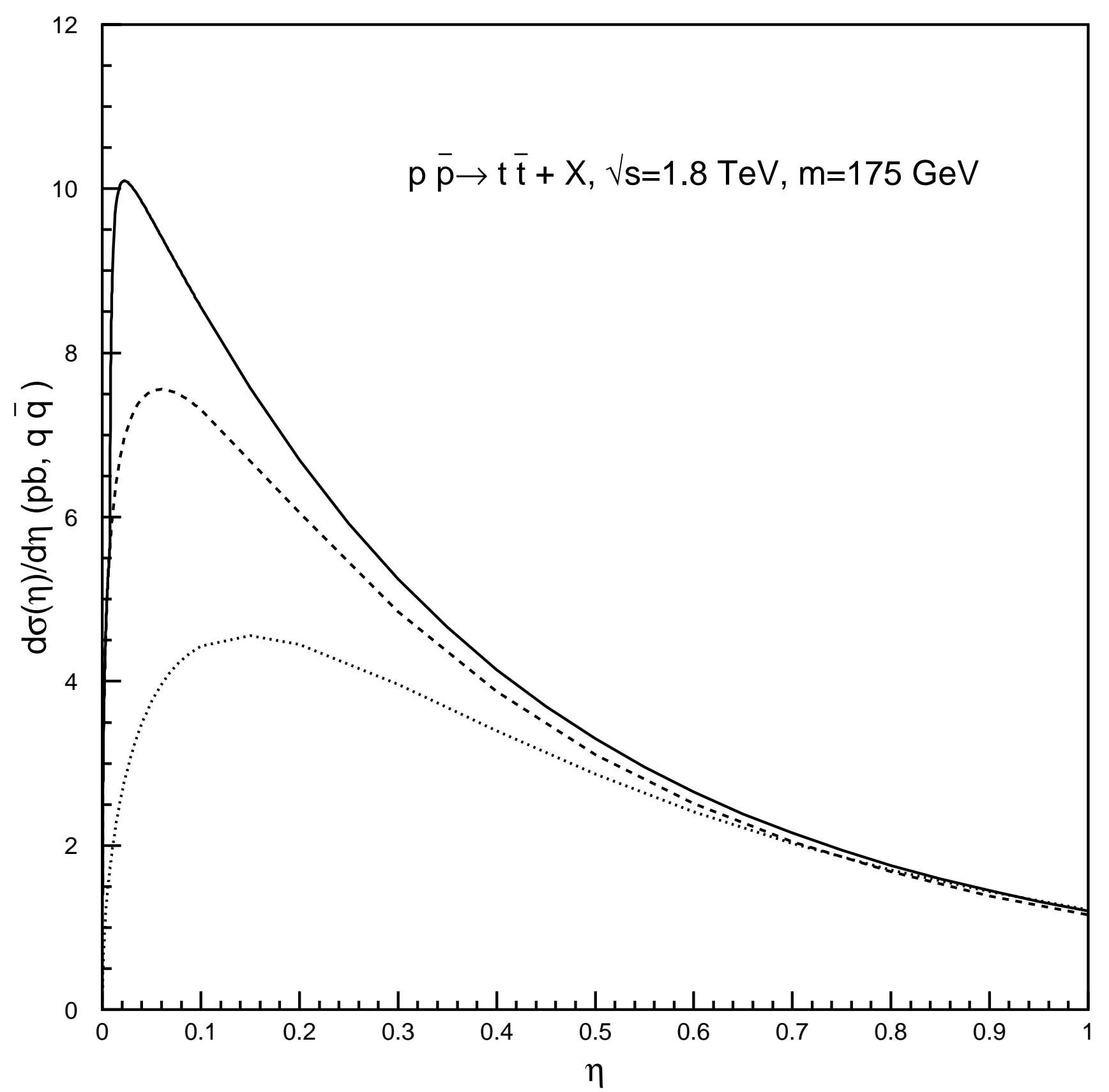

Figure $3 a$ 


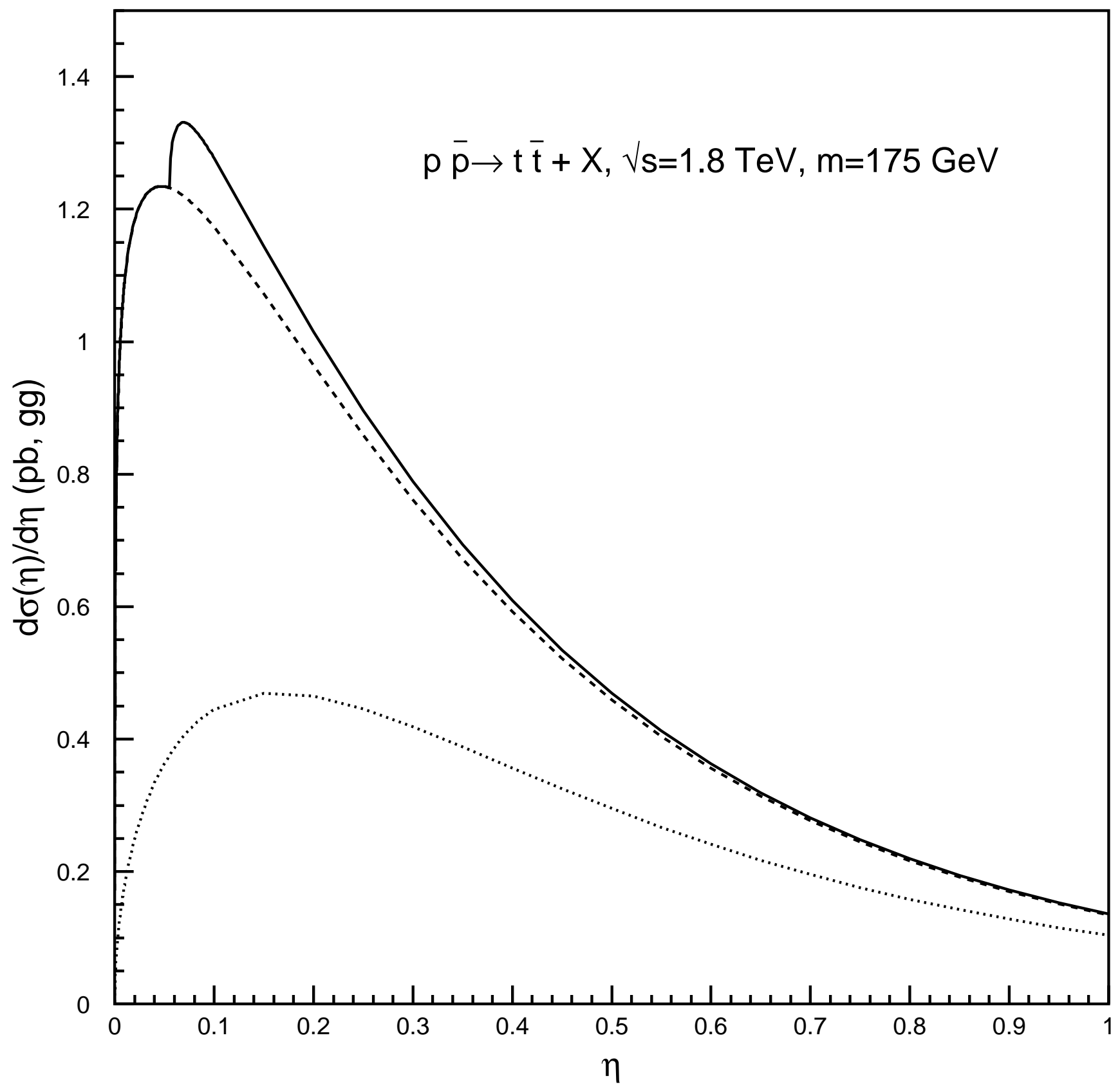

Figure $3 b$ 


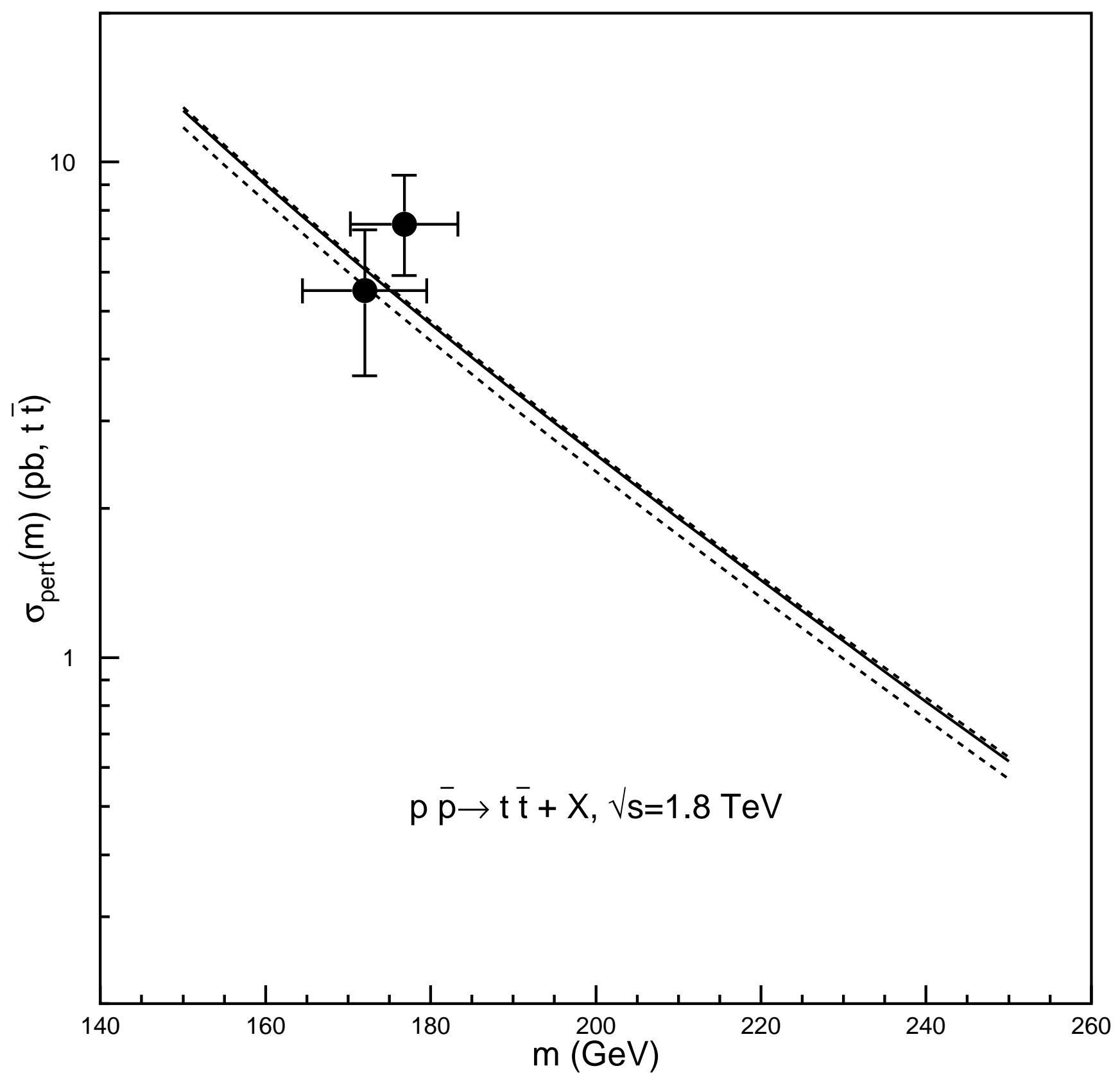

Figure 4 


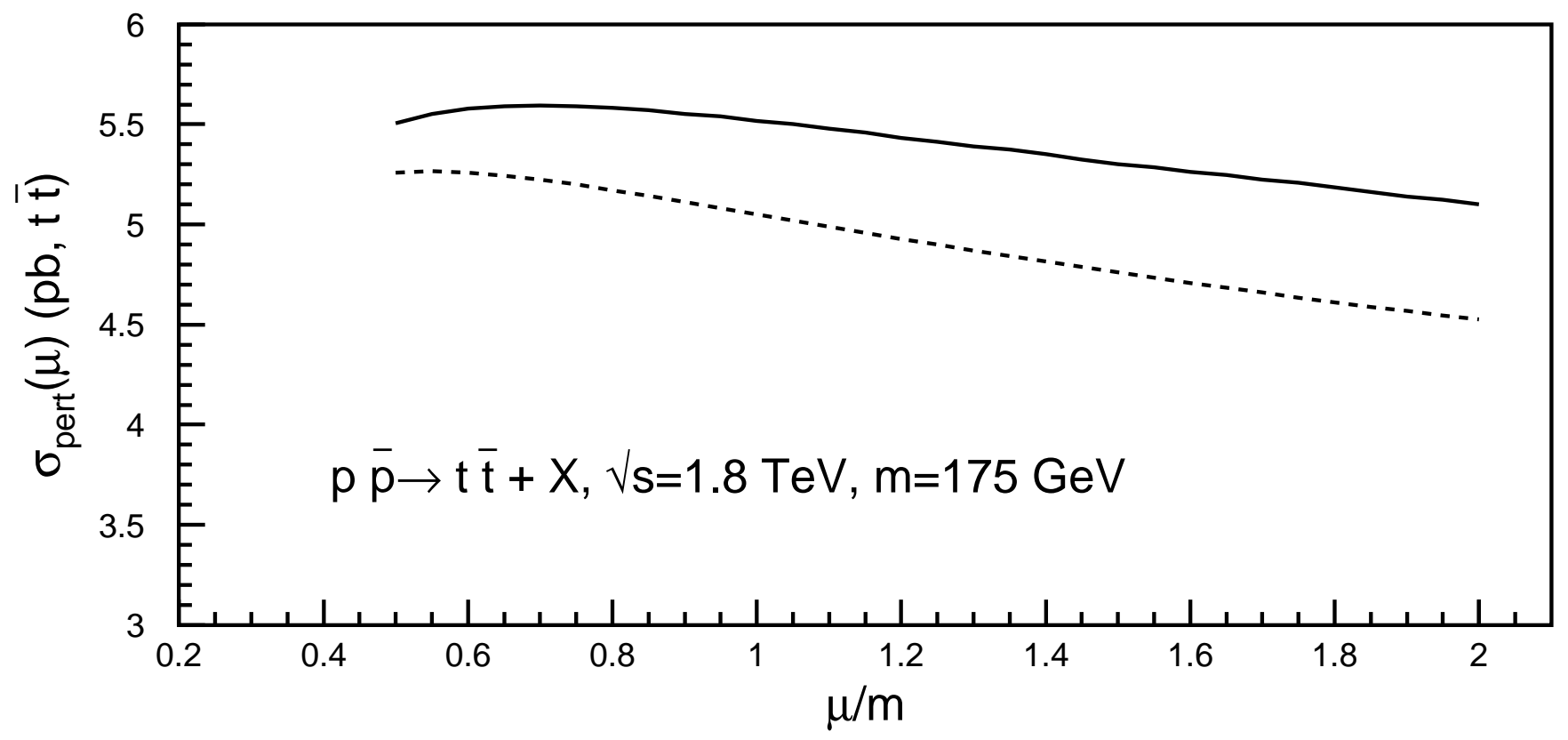

Figure 5 


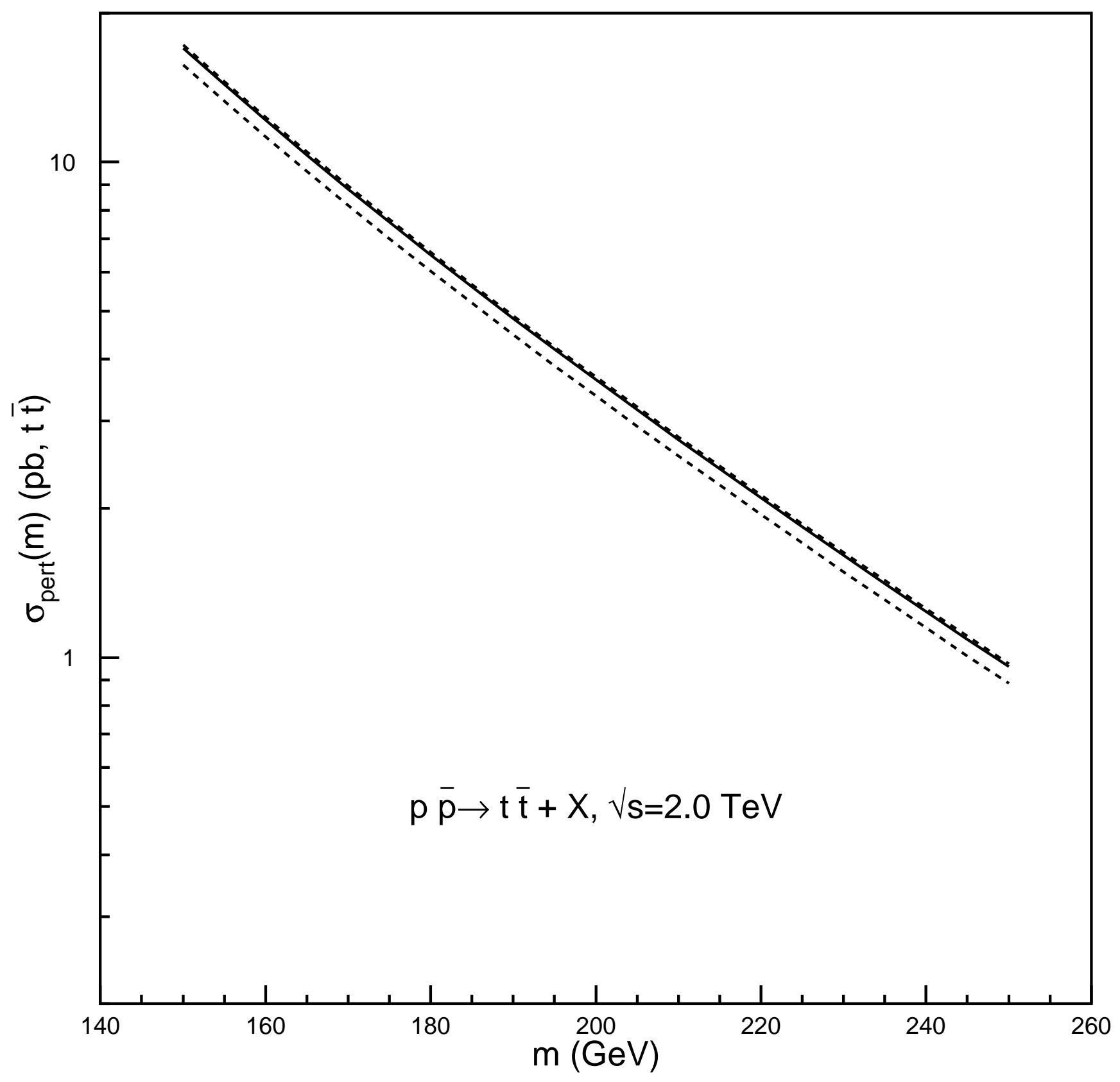

Figure 6 